\title{
Modelling and Analysis of Factors Affecting Moist Air Condensation on Mesh Like Surface for Sustainable Water Harvesting
}

\section{DOI : 10.36909/jer.ICCEMME.15779}

\author{
Punj Lata Singh* and B. S. Sikarwar \\ Department of Mechanical Engineering, Amity University Uttar Pradesh, Noida (UP), India \\ *Email: plsingh@amity.edu; Corresponding author
}

\begin{abstract}
Moist air condensation is a complex process because it relies on numerous significant parameters such as physio-chemical properties of a surface, thermal-physical properties of the moist air, and environmental conditions. Thus, the manuscript intent to structure the modelframework of sustainable water harvesting based on moist air condensation on mesh-like surfaces to increase the availability of potable water. Hence, the significant key factors related to sustainable water harvesting via condensation are identified from extant literature, consultation from researchers, and practitioners and subsequently confirmed by fabricating the experimental setup. Further than, an attempt by applying Total Interpretive Structural Modelling (TISM) is attained to provide a model framework. Thereafter, the procedure for analysis using MICMAC is implemented which performed to categorize the identified factors into the distinct clusters' dependent on their driving and dependence powers to justify their interrelationship with one another. The research reveals that surface coating, mesh geometry, and material of condensing surface are the most dominant factor in the required hierarchy which helps in improving the condensation rate. Thus, the prioritization of the factors with improvisation intends to fill the gap for scalable, economic, sustainable, and environmentfriendly condensation in various fields. This research work can be benefited to the industry to the area of water harvesting and energy conservation. Also, the researchers can consider the most influential factors identified in this research while fabricating experimental setups.
\end{abstract}

Keywords: Condensation; Factors; Total Interpretative Structure Modelling Approach; MICMAC analysis, Water harvesting

\section{INTRODUCTION}

In the existing scenario, life sustainability is possible because of water availability but the day by day increasing paucity of potable water has posed a serious risk for the global system. It has been noticed that the injudicious usage of water has created a challenge to counter the deficiency of drinkable water (Das, 2018). Though the land is already consisting of an ample amount of water in different form such as frozen glaciers, sea, aquifers, etc. The main concern lies with the actual extraction process using an economical modernized technique to meet the volatile demand of water (Manju and Sagar, 2017). Although, there exist various methods of obtaining potable water via harvesting (Tu et al., 2018) but extraction from atmospheric moist air is an extensively employed method due to its availability and cost (Fessehaye et al., 2014). However, the atmospheric moist air is a worthy alternative resource of water harvesting which considered to be consisting of approximately 6 times of all the river water resources on earth (Li et al., 2018). Thus, the world is attempting to arrange potable water resources by 
implementing several advanced technical practices to efficiently optimize the efficacious usage of water.

Many authors reported different harvesting methods of procuring moist air mainly by cooling condensation (Zhou et al., 2020). However, the cooling condensation is a completely an experimental and practically scalable method (Preston et al., 2015).

Considerably, it is also influenced by various parameters and equipment, which are adhered to. The phenomenon of condensation becomes evident when a cold surface possessing water repellence laid to humid environment (Shourideh et al., 2018). The condensation can be carried out either dropwise or film-wise and preferably the dropwise condensation due to the less thermal restraint to heat transfer is found more pertinent over film-wise condensation (Sikarwar et al., 2011). Eventually, dropwise condensation of moist air is an economical technique for efficient and effective water generation (Boreyko and Chen, 2009). The moist air condenses to the water usually occurs nearby a cool surface and is in form of dew which is affected by several factors (Cui et al., 2017). Thus, to attain the best efficiency, dropwise condensation progress depends upon the distinct factors correlated with it. Thus, a condensation process should be carried out in such a manner that the dependent and independent enhancement of factors should proceed on a hierarchical methodology. To achieve the required actions, for the betterment of fabrication of economical experimental setup, factors and enabling parameters are focused to execute the sustainable framework. Researchers and practitioners have reported the various factors associated with fundamental mechanisms, material, and methods for improving the heat transfer.

In summary, the factors standing responsible for moist air condensation on the selected mesh-like surface are required to identify. The prioritization of these factors from different perspectives will help the scientists and researchers to focus on few important factors to enhance the condensation phenomenon from moist air. The framework proposed by this research can enable the researchers to improvise their model by considering this study as a benchmark. Thus, the focused objective of this paper is outlined as follows:

- Identification of the vital factors existing responsible for the condensation of moist air on a mesh-like surface.

- Establishment of a mutual relationship approaching extend to relative importance and interdependence of every factor by utilizing Total Interpretive Structural Modelling (TISM) technique.

- Examining the factors by using MICMAC analysis focusing on the dependence and driving power that affecting condensation of moist air.

The remainder portion of the manuscript is structured as follows: Section 2 defines about the identification of the key factors responsible for the condensation of moist air from the literature review. Section 3 describes the proposed method to implement with the research procedure. Further, section 4 points out the application of the TISM approach and MICMAC analysis followed by results and discussion in section 5. Section 6 presents managerial implications and conclusion.

\section{FACTORS IDENTIFICATION FOR CONDENSATION OF MOIST AIR}

The identification of condensation factors is outlined to fulfil the aforesaid objectives. For this a two-phase methodology is used which is as follows:

Phase 1: The preliminary searches for the factors begin with the work published between 200521 by various researchers on condensations of moist air and its performance was extensively reviewed. It has also been observed that several factors can contribute not merely to increasing the efficacy but performance of the system too. Since there was a gap in the earlier literature 
and researchers are unable to improvise the efficiency of the condensation setup. For this purpose, fabrication of an experimental setup was processed in phase 2 , this would help in determining the suitability of the factors identified.

Phase 2: The fabricated experimental setup for performing dropwise condensation as shown in Figure 1 has been reported by Lata and Singh, 2021. This experimental setup for condensation was started with the fabrication of surface for condensation. The condensing surface was preferred to be metallic which holds good conductivity. Thus, a tubular copper with $99.9 \%$ of purity was considered to transform into a mesh-like surface. To fabricate a meshlike surface, copper tubes were brazed vertically and horizontally one upon another and textured to low wettability. The condensing surface becomes functional when connected to resourced equipment of setup. The equipment of a setup was a moist air generating unit, a controlled chilled water bath, a hygrometer, a temperature measuring device, leakproof pipes, and a data acquisition subsystem. These apparatuses were connected directly and indirectly to pursue the condensation phenomenon. The phenomenon on condensing surface was performed inside a closed acrylic chamber which was visualized using a high-resolution camera (Phantom, LAB3a10) through a visible plain window of the condensing chamber. Meanwhile, the required moist air was released inside the acrylic chamber from the unit of moist air generator through the connecting pipe. To prevent leakage, leakproof pipes were arranged and used for circulating the cooling effect inside the condensing surface. The cooling effect was accessed by a controlled chilled water bath which pumps the entire flow of cool water throughout the condensing surface. Therefore, a phase change from vapor form to liquid was observed when the cooled condensing surface was exposed to the moist air.

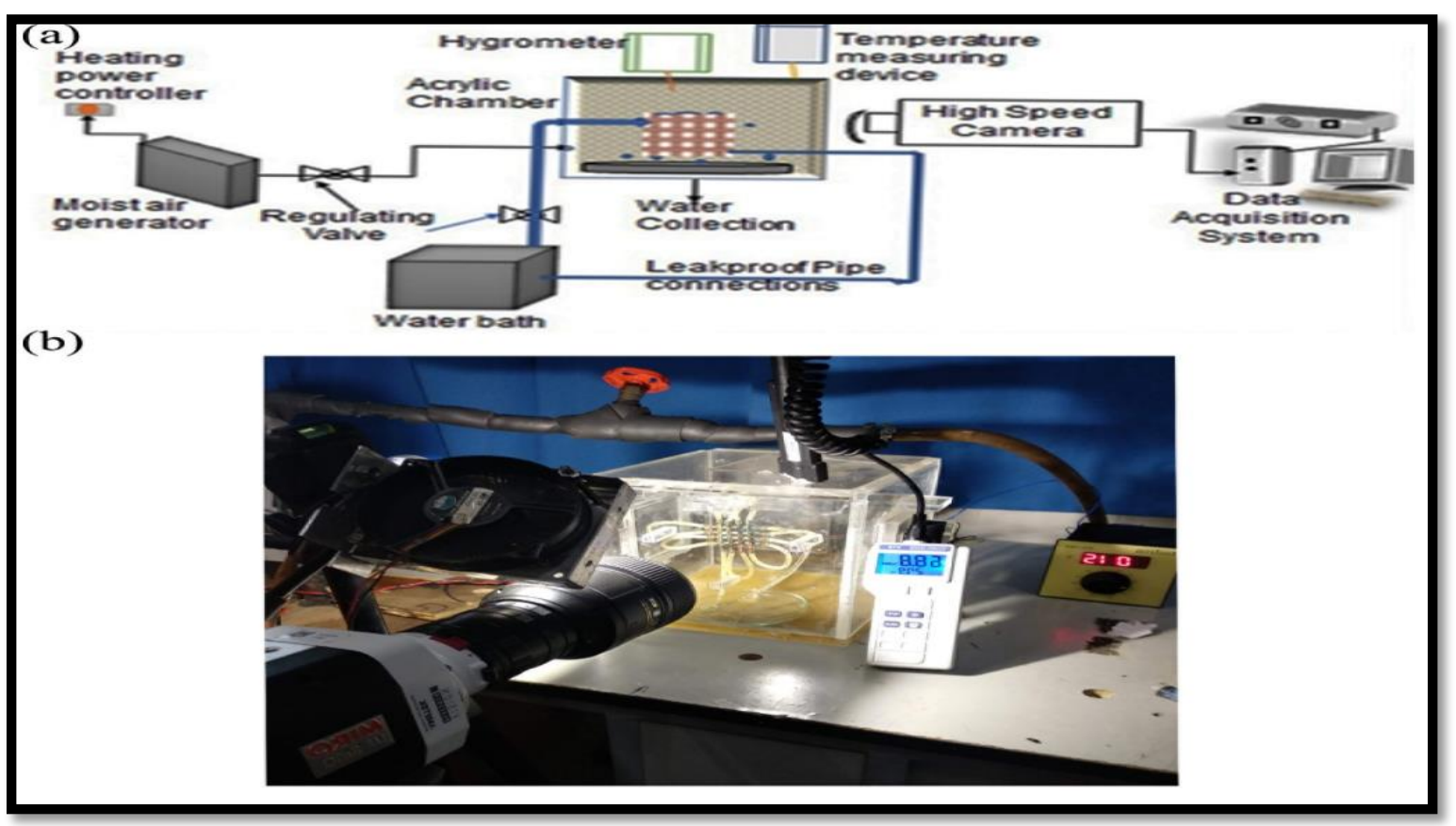

Figure 1 (a) Schematic diagram of an experimental setup for moist air condensation, (b) photograph of experimental apparatus

Thereby, the identified factors in phase 1 were validated from the experimental work performed in phase 2. Henceforth, it is analyzed and observed that the subsequent eleven enabling factors of condensation are accountable for enhancing the efficacy of the rate of condensation as shown in table 1 . 
Table 1 Factors affecting condensation

\begin{tabular}{|c|c|c|}
\hline Factors & Factors Description & References \\
\hline CF 1 & Surface coating & $\begin{array}{c}\text { Gou and Guo (2020); Kim et al., (2017); } \\
\text { Torresin et al., (2013) }\end{array}$ \\
\hline CF 2 & Mesh Geometry & Singh and Sikarwar (2020); Wen et al., (2018) \\
\hline CF 3 & Mesh Material & Seo et al., (2016); Xie et al., (2017) \\
\hline CF 4 & Relative Humidity & Jarimi et al., (2020); Castillo et al., (2015) \\
\hline CF 5 & Water Temperature & Grooten and Geld (2011); El Fil et al., (2020) \\
\hline CF 6 & Moist Air Speed & Kim et al., (2017); Yang et al., (2017) \\
\hline CF 7 & Water Speed & Torresin et al., (2013); Lata and Singh (2021) \\
\hline CF 8 & Thermal Conductivity & Warsinger et al., (2015); Patankar (2010) \\
\hline CF 9 & Droplet Dynamics & Niu and Tang (2014), Wen et al., (2016) \\
\hline CF 10 & Chambers Material & Yang et al., (2017) \\
\hline CF 11 & Leak-proof & Perujo (2005), Chen et al., (2011) \\
& Connections & \\
\hline
\end{tabular}

\section{METHODOLOGY}

The research methodology adopted here is beginning with identification of the factors through relevant analysis from literature and experimental setup and then, modeling them with the Multi Criteria Decision Making (MCDM) approach.

The procedure to complete the next objective of the research, the study is continued into twophase solution methodology.

Phase 1: Modelling the factors affecting condensation of moist air by TISM is a part of multi criteria decision making approach.

Phase 2: Analysis of driving and dependent factors by MICMAC (Matrice d'Impacts CroisesMultipication Applique an Classment) analysis. Figure 2 depicts a research methodology followed to evaluate the condensation factors.

In the existing study, the TISM approach is implemented profoundly to develop the model. This approach is an upgraded form of technique interpretive structural modelling (Sushil, 2017). It is a methodology that provides the contextual relationships among factors related to the system (Raj et al., 2008) which is used by various practitioners in a different field such as agility manufacturing systems (Sindhwani and Malhotra, 2017), incorporation of agile, lean, and green manufacturing (Sindhwani et al., 2019). TISM is a refined version of ISM used and contributed to the numerous studies. However, many authors in the various fields such as in cloud computation (Sagar et al., 2013), pharmacological (Wasuja and Sagar, 2012), manufacturing system for flexibility (Sushil, 2017) etc. has been effectively used TISM approach. The flow diagram of TISM approach is represented in Figure 3. 


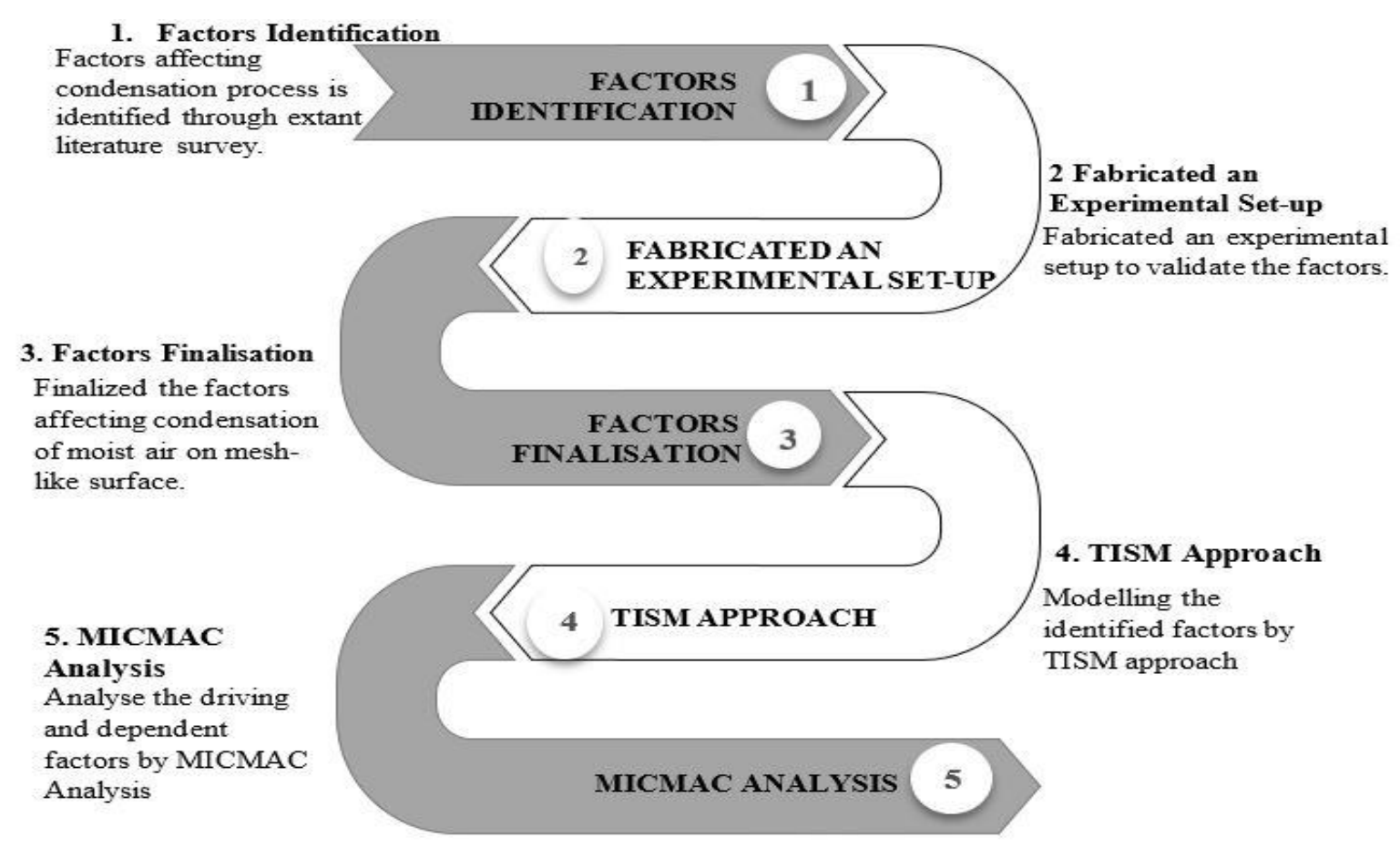

Figure 2 Research Methodology

\section{Factors identification}

The factors identification work was executed and completed carefully by referring to the reputed journals which were verified after experiment performed explained systematically in this study. There are eleven factors identified and verified are enlisted as mentioned in table 1.

\section{Contextual relationship development}

The procedure of TISM begins with the requirement to develop an appropriate relationship between identified factors. This relationship can be ' $X$ should oppose or influence $Y$ ' or ' $Y$ should oppose or influence on $X^{\prime}$ ' in the moist air condensation of water vapor of on a meshlike surface. To obtain the contextual relationship between factors, data was collected in the above stage.

\section{Relationship Interpretation}

The step describes the functional working methodology of the inter-relationship between factors. In this stage, a description about the factors either opposing or influencing each other is compiled.

\section{Comparison pairwise}

In order to perform comparison, the first factor is compared to all mentioned factors with individual row. For comparing individual factor, it must be scripted to either Yes (Y) or No (N). After comparing all factors, a relationship in the form $\mathrm{Y}$ or $\mathrm{N}$ is tabulated in table 2. 


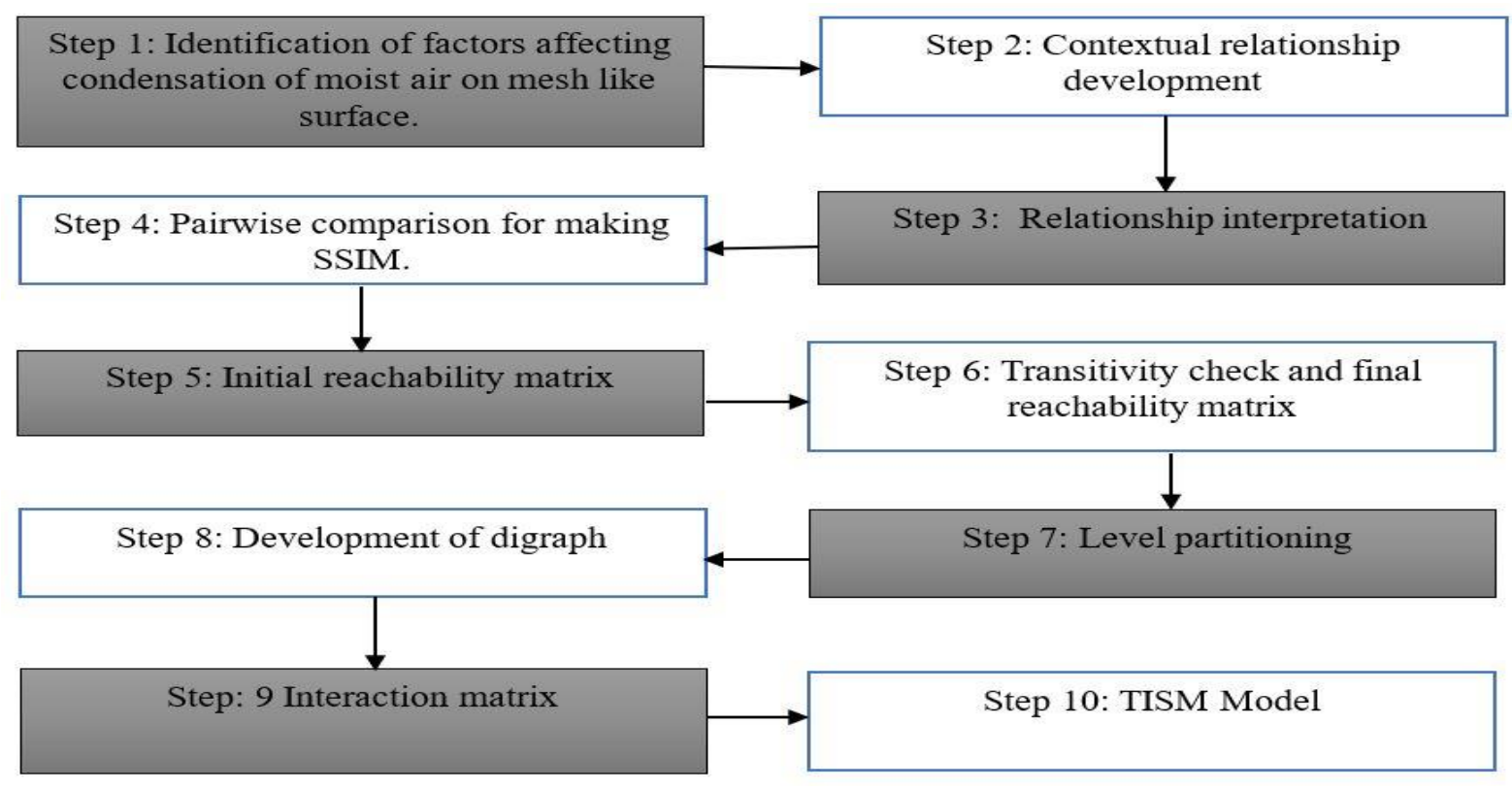

Figure 3 Flow diagram for TISM approach

\section{Initial matrix for reachability}

This step changed the cell which is represented with ' $\mathrm{Y}$ ' is reformed with ' 1 ' and the cell represented with ' $\mathrm{N}$ ' is reformed with ' 0 '.

Table 2 Structured Self Interaction Matrix

\begin{tabular}{|c|c|c|c|c|c|c|c|c|c|c|c|}
\hline Factors & F1 & F2 & F3 & F4 & F5 & F6 & F7 & F8 & F9 & F10 & F11 \\
\hline F1 & Y & N & N & N & N & N & N & Y & Y & N & N \\
\hline F2 & N & Y & N & N & Y & Y & Y & Y & Y & N & Y \\
\hline F3 & N & Y & Y & Y & Y & N & N & Y & Y & N & N \\
\hline F4 & N & N & N & Y & Y & N & N & Y & Y & N & N \\
\hline F5 & N & N & N & N & Y & N & N & Y & Y & N & Y \\
\hline F6 & N & N & N & Y & Y & Y & N & N & Y & N & Y \\
\hline F7 & N & N & Y & N & N & N & Y & N & Y & N & Y \\
\hline F8 & N & N & Y & N & Y & N & N & Y & Y & N & Y \\
\hline F9 & N & N & N & N & N & N & N & N & Y & N & N \\
\hline F10 & N & N & N & N & N & N & N & N & N & Y & N \\
\hline F11 & N & Y & N & Y & N & N & N & Y & Y & N & Y \\
\hline
\end{tabular}

\section{Transitiveness and Reachability Matrix (RM)}

Now, in this step the obtained initial RM has undergone checking for transitivity. It holds a relation among three different factors in a such a way that if a relation exists between the $X$ and $\mathrm{Y}$ and then a relation exists between $\mathrm{Y}$ and $\mathrm{Z}$ therefore, automatically a relation should exist among $\mathrm{X}$ and $\mathrm{Z}$. Now, using the concept final $\mathrm{RM}$ is achieved by including the transitiveness. Hence, the transitiveness is marked as $1 *$ in the final RM as represented in table 3. 
Table 3 Final Reachability Matrix

\begin{tabular}{|c|c|c|c|c|c|c|c|c|c|c|c|}
\hline Factors & F1 & F2 & F3 & F4 & F5 & F6 & F7 & F8 & F9 & F10 & F11 \\
\hline F1 & 1 & 0 & $1^{*}$ & 0 & $1^{*}$ & 0 & 0 & 1 & 1 & 0 & $1^{*}$ \\
\hline F2 & 0 & 1 & $1^{*}$ & $1^{*}$ & 1 & 1 & 1 & 1 & 1 & 0 & 1 \\
\hline F3 & 0 & 1 & 1 & 1 & 1 & $1^{*}$ & $1^{*}$ & 1 & 1 & 0 & $1^{*}$ \\
\hline F4 & 0 & 0 & $1^{*}$ & 1 & 1 & 0 & 0 & 1 & 1 & 0 & $1^{*}$ \\
\hline F5 & 0 & $1^{*}$ & $1^{*}$ & $1^{*}$ & 1 & 0 & 0 & 1 & 1 & 0 & 1 \\
\hline F6 & 0 & $1^{*}$ & 0 & 1 & 1 & 1 & 0 & $1^{*}$ & 1 & 0 & 1 \\
\hline F7 & 0 & $1^{*}$ & 1 & $1^{*}$ & $1^{*}$ & 0 & 1 & $1^{*}$ & 1 & 0 & 1 \\
\hline F8 & 0 & $1^{*}$ & 1 & $1^{*}$ & 1 & 0 & 0 & 1 & 1 & 0 & 1 \\
\hline F9 & 0 & 0 & 0 & 0 & 0 & 0 & 0 & 0 & 1 & 0 & 0 \\
\hline F10 & 0 & 0 & 0 & 0 & 0 & 0 & 0 & 0 & 0 & 1 & 0 \\
\hline F11 & 0 & 1 & $1^{*}$ & 1 & $1^{*}$ & $1^{*}$ & $1^{*}$ & 1 & 1 & 0 & 1 \\
\hline
\end{tabular}

\section{Level partitioning}

A partition of levels is executed by finding the antecedent and reachability sets for all the factors. After this, another column of intersection set is made that contains the common factors present in antecedent and reachability sets. Hence, it turned to be removed and proceed similarly, after further iterations is tabulated in table 4.

Table 4 Level Partition

\begin{tabular}{|c|l|l|l|l|}
\hline Factors & Reachability Set & \multicolumn{1}{|c|}{ Antecedent Set } & \multicolumn{1}{c|}{ Intersection Set } & Level \\
\hline $\mathbf{1}$ & 1 & 1 & 1 & V \\
\hline $\mathbf{2}$ & $2,3,6,7$ & $2,3,6,7$ & $2,3,6,7$ & III \\
\hline $\mathbf{3}$ & 3 & 3 & 3 & IV \\
\hline $\mathbf{4}$ & $3,4,5,8,11$ & $2,3,4,5,6,7,8,11$ & $3,4,5,8,11$ & II \\
\hline $\mathbf{5}$ & $2,3,4,5,8,11$ & $1,2,3,4,5,6,7,8,11$ & $2,3,4,5,8,11$ & II \\
\hline $\mathbf{6}$ & 2,6 & $2,3,6$ & 2,6 & III \\
\hline $\mathbf{7}$ & $2,3,7$ & $2,3,7$ & $2,3,7$ & III \\
\hline $\mathbf{8}$ & $2,3,4,5,8,11$ & $1,2,3,4,5,6,7,8,11$ & $2,3,4,5,8,11$ & II \\
\hline $\mathbf{9}$ & 9 & $1,2,3,4,5,6,7,8,9,11$ & 9 & I \\
\hline $\mathbf{1 0}$ & 10 & 10 & 10 & I \\
\hline $\mathbf{1 1}$ & $2,3,4,5,6,7,8,11$ & $1,2,3,4,5,6,7,8,11$ & $2,3,4,5,6,7,8,11$ & II \\
\hline
\end{tabular}

\section{Development of digraph}

In this step, the relationship between the links is represented on the graph as per the final RM.

\section{Interaction matrix (IM)}

After the diagraph, the changing of entire interaction entries of 1 thus representing in a matrix. Now, the knowledge base technique is used to check the all ' 1 ' entry in the IM. 


\section{Total Interpretative Structural Modelling (TISM)}

This step comprises with the support of digraph and IM. Nodes of digraph are switched with the explanation of factors then finally the total interpretive structural model is represented in Figure 4.

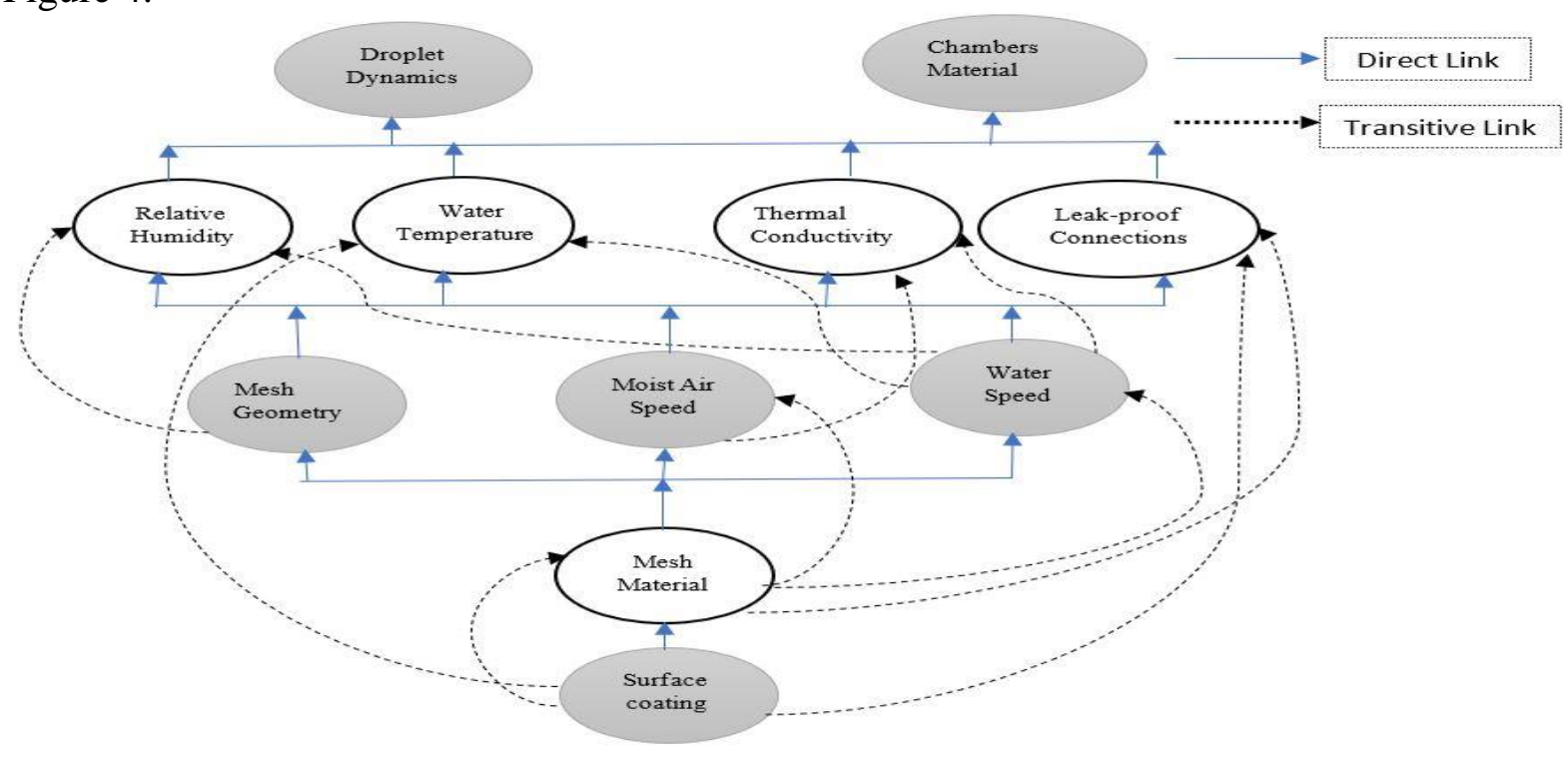

Figure 4 TISM Model

\section{MICMAC Analysis}

Three key functions are performed by MICMAC analysis i.e. identifying relevant factors and building relationships between the factors. It helps in identifying the vital factors that systematically drives the system in each category. Subsequently, the table which is established using the final RM where sum of all rows is drive power and those of columns is dependence power. Using interaction matrix, a grid is plotted as shown in Figure 5, and results inferred from it.

\section{RESULTS AND DISCUSSION}

It is observed from literature, that many researchers are working on mesh- like surface for moist air condensation, but it is very difficult to identify among factors about their important role in this respective area. All research has covered various experiments but many of the experiments still lacks to provide optimum results. So, the diverse factors collected from literature and experiments have been inserted into the total interpretive structural modeling for relationship identification. Finally, the result is categorized in four parts such as: dependent factors, autonomous factors, independent factors, and linkage factors.

Quadrant-I (Autonomous factors): These factors possess a feeble dependent and driving power. As per the paper's results, only one factor falls under this category i.e., chamber material (CF10). 


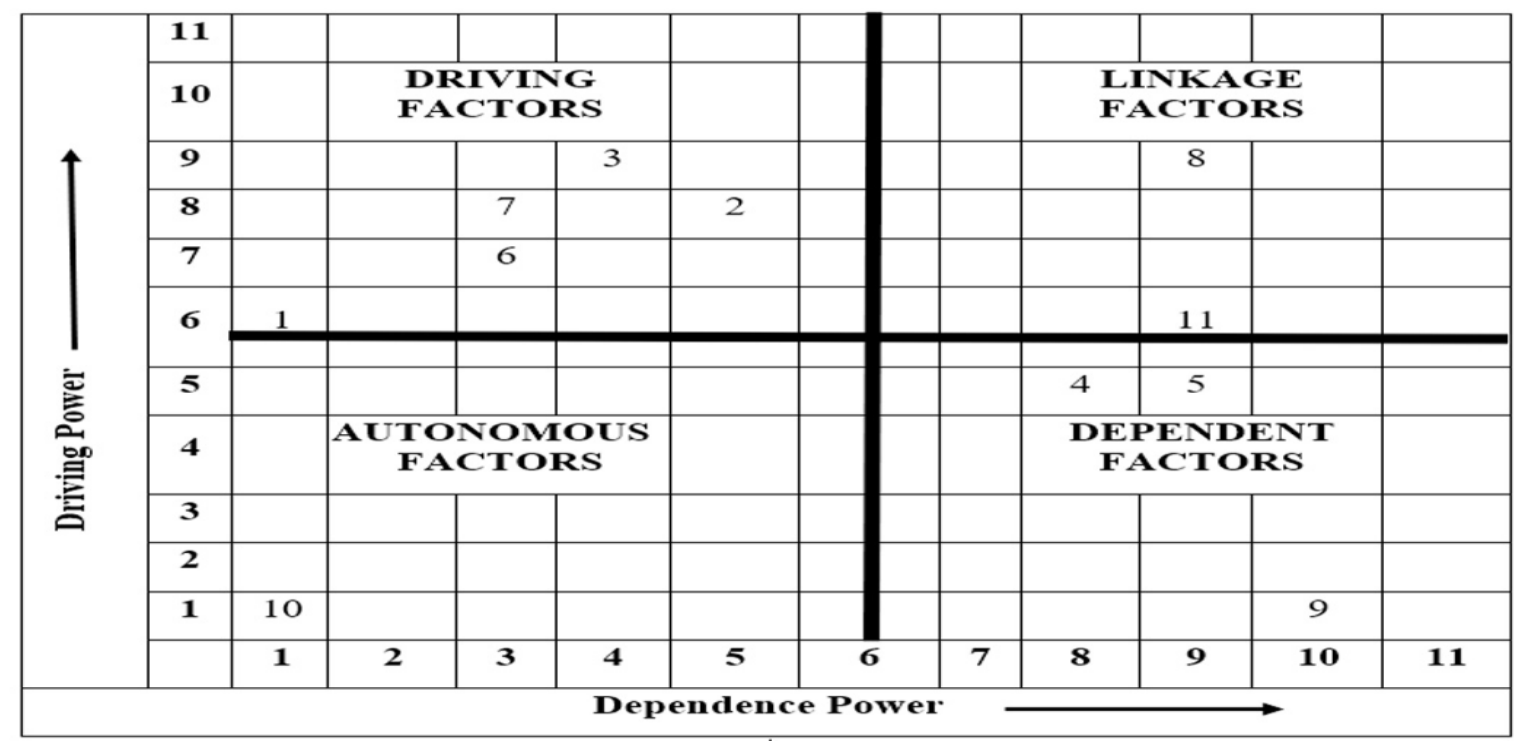

Figure 5. MICMAC Analysis

Quadrant-II (Dependent factors): The factors in this quadrant establish a very weak driving but a strong dependent power among one another. After the study, three factors are lying under this quadrant i.e., droplet dynamics (CF9), relative humidity (CF4), water temperature (CF5). Out of these three two factors, CF4 and CF5 are lying on the borderline of dependent and linkage factors.

Quadrant-III (Linkage factors): The factors in this quadrant upholds a strong driving as well strong dependence ability. The performed action on the evaluated factors, influence the others and would provide an effective response. They can disturb the whole system. Thermal Conductivity (CF8) and Leak-proof Connections (CF11) factors lie under this category. It means if proof connections are not perfectly obtained then it can disturb the whole system. From this study, one of the factors thermal conductivity is also playing a very important for making a perfect system. According to independent factors (Quadrant-IV), these region factors get less dependency and high driving power and the result from Figure 5 inferred that Surface coating (CF1), Mesh Geometry (CF2), Mesh Material (CF3), Moist Air Speed (CF6), Water Speed (CF7) are coming under this quadrant. Thus, this analyzed study shows that these affecting factors may be treated as "key factors". All factors that come under this quadrant act as an essential part in the condensation process. It reveals the researcher needs to aim on mesh geometry (CF2), mesh material (CF3), and water speed factors (CF7) on priority as these factors influences high driving and less dependence power. Afterwards, we need to focus on Moist Air Speed (CF6) means control on moist airspeed can play a vital role in the condensation process. Lastly, Surface coating (CF1) factor lies under this quadrant, this factor also serves to be a good driving factor. All the factors in this quadrant, jointly help the researchers in increasing the moist air condensation on the surfaces like mesh.

\section{CONCLUSION}

This influential study explores the importance of condensation in countering one of the extremely crucial crisis that the World is facing today. The research work contributes to the

investigation of eleven key factors which directly or indirectly affects the condensation for the mesh-like surface. The model for the condensation process on the mesh-like surface has 
established by applying TISM and MICMAC analysis. Finally, it can be applied to fabricate a suitable experimental setup for the condensation process. This finding will allow researchers, practitioner, and application-based industries to efficiently use their resources while selecting the appropriate and most significant factor for optimization of condensation process. These results will emphasize in understanding the factors that entirely influencing the condensation setups. The researchers and practitioners will also achieve a feasible and sustainable solution to improved condensation rate from the above findings in terms of competitiveness, readily, and environmental concern, etc.

A further identification of factors with the help of different experimentation, academia and industry experts, researchers, practitioners etc. can provide more help in improving the condensation phenomenon. Furthermore, MCDM techniques can be implemented on identified factors for better understanding of the condensation process.

The research work outlook obtained result which provides following actions to foster the sustainable condensation phenomenon.

i. This study expresses that mesh geometry is playing a very significant role in the condensation process. In experiments, mesh geometry acts as bridging between water repellent surface and high-performance vapor-liquid phase change. On this consideration, the fine to finer selection of structured mesh can provide an accurate and precise condensation surface.

ii. The probable feasibility of the condensation procedure is associated with the material of mesh and nucleates drops beneath the temperature of dew point. If the material of mesh is metallic, it enhances the conductivity which preserves the functional property of condensing surface and progress in phase change. Thus, the appropriate selection of mesh material will surely help the researchers in improvising the condensation rate.

iii. The homogeneous coating on the surface enables low wettability and increases the sliding of drop which directly enhances the condensation rate. The surface coating stimulates durability as well as productivity. Thus, improvising the surface coating will further extends to economical condensation performance.

\section{ACKNOWLEDGMENTS}

The authors acknowledge the fund support from the Science and Engineering Research Board (SERB), India, established through an Act of Parliament: SERB Act 2008, Department of Science \& Technology (DST), India, Government of India (Project no: ECR/2016/000020). The authors acknowledge expert's opinion for contributing their efforts, support, and time as input for the study of this research work.

\section{REFERENCES}

1. Das, S. D. (2018). Assessment of atmospheric water generator for rural and remote India. IOSR Journal of Electrical and Electronics Engineering (IOSR-JEEE), 13(2): 67-74.

2. Manju, S., \& Sagar, N. (2017). Renewable energy integrated desalination: A sustainable solution to overcome future fresh-water scarcity in India. Renewable and Sustainable Energy Reviews, 73: 594-609.

3. Tu, Y., Wang, R., Zhang, Y., \& Wang, J. (2018). Progress and expectation of atmospheric water harvesting. Joule, 2(8): 1452-1475. 
4. Fessehaye, M., Abdul-Wahab, S. A., Savage, M. J., Kohler, T., Gherezghiher, T., \& Hurni, H. (2014). Fog-water collection for community use. Renewable and Sustainable Energy Reviews, 29: 52-62.

5. Li, R., Shi, Y., Shi, L., Alsaedi, M., \& Wang, P. (2018). Harvesting water from air: using anhydrous salt with sunlight. Environmental science \& technology, 52(9): 5398-5406.

6. Zhou, X., Lu, H., Zhao, F., \& Yu, G. (2020). Atmospheric water harvesting: a review of material and structural designs. ACS Materials Letters, 2(7): 671-684.

7. Preston, D. J., Mafra, D. L., Miljkovic, N., Kong, J., \& Wang, E. N. (2015). Scalable graphene coatings for enhanced condensation heat transfer. Nano letters, 15(5): 2902-2909.

8. Shourideh, A. H., Ajram, W. B., Al Lami, J., Haggag, S., \& Mansouri, A. (2018). A comprehensive study of an atmospheric water generator using Peltier effect. Thermal Science and Engineering Progress, 6: 14-26.

9. Sikarwar, B. S., Battoo, N. K., Khandekar, S., \& Muralidhar, K. (2011). Dropwise condensation underneath chemically textured surfaces: simulation and experiments. Journal of Heat Transfer, 133(2).

10. Boreyko, J. B., \& Chen, C. H. (2009). Self-propelled dropwise condensate on superhydrophobic surfaces. Physical review letters, 103(18): 184501

11. Cui, Y., Li, D., \& Bai, H. (2017). Bioinspired smart materials for directional liquid transport. Industrial \& Engineering Chemistry Research, 56(17): 4887-4897.

12. Singh, P. L., \& Sikarwar, B. S. (2021). Assessment of Mesh-Like Surface for Water Harvesting from Moist Air. In Advances in Systems Engineering (pp. 161-168). Springer, Singapore.

13. Gou, X., \& Guo, Z. (2020). Facile Fabrication of Slippery Lubricant-Infused CuO-Coated Surfaces with Different Morphologies for Efficient Water Collection and Excellent Slippery Stability. Langmuir, 36(30): 8983-8992.

14. Kim, H., Yang, S., Rao, S. R., Narayanan, S., Kapustin, E. A., Furukawa, H., ... \& Wang, E. N. (2017). Water harvesting from air with metal-organic frameworks powered by natural sunlight. Science, 356(6336): 430-434.

15. Torresin, D., Tiwari, M. K., Del Col, D., \& Poulikakos, D. (2013). Flow condensation on copper-based nanotextured superhydrophobic surfaces. Langmuir, 29(2): 840-848.

16. Singh, P. L., \& Sikarwar, B. S. (2020). Condensation of Moist Air on Mesh-like Surfaces. Asian Journal of Water, Environment and Pollution, 17(2): 65-72.

17. Wen, R., Xu, S., Zhao, D., Yang, L., Ma, X., Liu, W., ... \& Yang, R. (2018). Sustaining enhanced condensation on hierarchical mesh-covered surfaces. National Science Review, 5(6): 878-887

18. Seo, D., Lee, J., Lee, C., \& Nam, Y. (2016). The effects of surface wettability on the fog and dew moisture harvesting performance on tubular surfaces. Scientific reports, 6(1): 1-11.

19. Xie, J., Xu, J., He, X., \& Liu, Q. (2017). Large scale generation of micro-droplet array by vapor condensation on mesh screen piece. Scientific reports, 7(1): 1-13.

20. Jarimi, H., Powell, R., \& Riffat, S. (2020). Review of sustainable methods for atmospheric water harvesting. International Journal of Low-Carbon Technologies, 15(2): 253-276.

21. Castillo, J. E., Weibel, J. A., \& Garimella, S. V. (2015). The effect of relative humidity on dropwise condensation dynamics. International Journal of Heat and Mass Transfer, 80:759-766

22. Grooten, M. H. M., \& Van der Geld, C. W. M. (2011). Dropwise condensation from flowing air-steam mixtures: Diffusion resistance assessed by controlled drainage. International Journal of Heat and Mass Transfer, 54(21-22): 4507-4517.

23. El Fil, B., Kini, G., \& Garimella, S. (2020). A review of dropwise condensation: Theory, modeling, experiments, and applications. International Journal of Heat and Mass Transfer, 160: 120172 
24. Kim, M. H., Kim, H., Lee, K. S., \& Kim, D. R. (2017). Frosting characteristics on hydrophobic and superhydrophobic surfaces: A review. Energy Conversion and Management, 13:, 1-11.

25. Yang, K. S., Lin, K. H., Tu, C. W., He, Y. Z., \& Wang, C. C. (2017). Experimental investigation of moist air condensation on hydrophilic, hydrophobic, superhydrophilic, and hybrid hydrophobic-hydrophilic surfaces. International Journal of Heat and Mass Transfer, 115: 1032-1041.

26. Warsinger, D. E., Swaminathan, J., \& Maswadeh, L. A. (2015). Superhydrophobic condenser surfaces for air gap membrane distillation. Journal of Membrane Science, 492: 578587.

27. Patankar, N. A. (2010). Supernucleating surfaces for nucleate boiling and dropwise condensation heat transfer. Soft Matter, 6(8): 1613-1620.

28. Niu, D., \& Tang, G. H. (2014). Static and dynamic behavior of water droplet on solid surfaces with pillar-type nanostructures from molecular dynamics simulation. International Journal of Heat and Mass Transfer, 79: 647-654.

29. Wen, R., Lan, Z., Peng, B., Xu, W., Ma, X., \& Cheng, Y. (2016). Droplet departure characteristics and dropwise condensation heat transfer at low steam pressure. Journal of Heat Transfer, 138(7).

30. Perujo, P. M. (2005). Condensation of Water Vapor and Acid Mixtures from Exhaust Gases.

31. Chen, X., Wu, J., Ma, R., Hua, M., Koratkar, N., Yao, S., \& Wang, Z. (2011). Nanograssed micropyramidal architectures for continuous dropwise condensation. Advanced functional materials, 21(24): 4617-4623.

32. Sushil. (2017). Multi-criteria valuation of flexibility initiatives using integrated TISM-IRP with a big data framework. Production Planning \& Control, 28(11-12): 999-1010.

33. Raj, T., Shankar, R., \& Suhaib, M. (2008). An ISM approach for modelling the enablers of flexible manufacturing system: the case for India. International Journal of Production Research, 46(24): 6883-6912.

34. Sindhwani, R., \& Malhotra, V. (2017). Modelling and analysis of agile manufacturing system by ISM and MICMAC analysis. International Journal of System Assurance Engineering and Management, 8(2): 253-263.

35. Sindhwani, R., Mittal, V. K., Singh, P. L., Aggarwal, A., \& Gautam, N. (2019). Modelling and analysis of barriers affecting the implementation of lean green agile manufacturing system (LGAMS). Benchmarking: An International Journal., 26(2): 498-529.

36. Sagar, M., Bora, S., Gangwal, A., Gupta, P., Kumar, A., \& Agarwal, A. (2013). Factors affecting customer loyalty in cloud computing: A customer defection-centric view to develop a void-in-customer loyalty amplification model. Global Journal of Flexible Systems Management, 14(3): 143-156.

37. Wasuja, S., \& Sagar, M. (2012). Cognitive bias in salespersons in specialty drug selling of pharmaceutical industry. International Journal of Pharmaceutical and Healthcare Marketing, 6(4): 310-335.

38. Dupperin, J. C., \& Godet M. (1973). Method De Hierar Chization Des Elements D'um System, Rapport Economique De CEA, R-45-51, Paris 1973. 